\begin{tabular}{|c|l|}
\hline Title & Interfacial electron transfer as a significant step in photoelectrochemical reactions on some semiconductors \\
\hline Author(s) & Bockris, J. O 'M.; U osaki, K.; Kita, H. \\
\hline Citation & $\begin{array}{l}\text { Journal of A pplied Physics, 52(2), 808-810 } \\
\text { https://doi.org/10.1063/1.328847 }\end{array}$ \\
\hline Issue Date & 1981-02 \\
\hline Doc URL & http://hdl.handle.net/2115/6127 \\
\hline Rights & Copyright $\odot$ 1981 A merican Institute of Physics \\
\hline Type & article \\
\hline File Information & JAP52-2.pdf \\
\hline
\end{tabular}

Instructions for use 


\title{
Interfacial electron transfer as a significant step in photoelectrochemical reactions on some semiconductors
}

\author{
J. O'M. Bockris
}

Department of Chemistry, Texas A\&M University, College Station, Texas 77843

K. Uosaki and H. Kita

Department of Chemistry, Hokkaido University, Sapporo, Japan

(Received 18 August 1980; accepted for publication 3 November 1980)

\begin{abstract}
Photoelectrochemical kinetics at the semiconductor-solution interface has been considered in all treatments in the literature, except one, to be rate controlled by processes inside the semiconductor. Evidence is presented which suggest that, at least for cathodic reactions on $p$-CdTe, the rate determining step is interfacial electron transfer, and a part of the total potential difference at the interface exists in the Helmholtz layer.
\end{abstract}

PACS numbers: $68.48 .+\mathrm{f}$

\section{INTRODUCTION}

In the treatment of electrochemical processes, one regards several steps as occuring consecutively, one of which is rate determining. This step may change with the potential, $p \mathrm{H}$, etc. The mechanism of photoelectrochemical reactions at semiconductor electrodes is not well understood. ${ }^{1}$ At sufficiently large potential biases (saturation region), the dependence of the photocurrent on potential must become controlled by a transport process of charge carriers to the interface. $^{2-4}$ At small potential biases (which may represent the significant region for practical devices), other processes than transport within the solid, in particular, the interfacial transfer of electrons from the semiconductor to acceptors in the solution, or vice versa, may become rate determining. In the literature, the assumption of most theories (but cf. Refs. 5, 6, and 9) is that processes within the solid control the photoelectrochemical rate. Here, it will be shown that interfacial charge transfer is rate determining for lower biases of $n$ CdTe photocathodes.

Experimental details of the work - one result of which is here interpreted - have been given in previous papers. ${ }^{6-7}$

\section{RESULTS}

The photocurrent-potential relations of $p$-type CdTe in 1-N NaOH under illumination of $900-\mathrm{W}$ Xe lamp are shown in Fig. 1. The existence of oxygen in the solution affected the shape of the $I-V$ curve radically. When oxygen was removed by bubbling with hydrogen for $30 \mathrm{~min}$., the photcurrent was only observed after the electrode potential became more negative than $-0.75 \mathrm{~V}$ (NHE), despite the positive flat band potential ( + $0.21 \mathrm{~V}$ ). However, when the experiment was carried out without removing oxygen, the photocurrent was observed at a potential as positive as $-0.1 \mathrm{~V}$ and reached the saturated photocurrent at around $-0.3 \mathrm{~V}$, then increased again from $-0.7 \mathrm{~V}$.

\section{DISCUSSION}

If in a cathodic reaction the supply of photoexcited electrons from the bulk to the semiconductor surface is the rate determining step, the photocurrent $i_{p}$ is given by

$$
i_{p} \alpha j_{p}=\int_{0}^{\infty} N_{p}(E) d E
$$

where $j_{p}$ is the number of photoexcited electrons arriving at the surface per unit time and area and $N_{p}(E)$ is the number of photoexcited electrons arriving at the surface per unit time per unit area, with energy between $E$ and $E+d E$. Energy levels are taken as zero at the bottom of the coduction band at the surface. According to this mechanism, the photocurrent should begin to be observed at a potential just negative (for a $p$-type electrode) to the flat band potential. An example where such a prediction holds well is $\mathrm{TiO}_{2} \cdot{ }^{8}$ Because $\mathrm{TiO}_{2}$ is an $n$-type semiconductor, the anodic photocurrent is observed at a potential just positive to the flat band potential. However, for $p$-CdTe, the potential at which the current is observed is not that of the flat band region. The photocurrent (Fig. 1) was observed at a potential at least $0.3 \mathrm{~V}$ negative to the flat band potential with $\mathrm{O}_{2}$ in the solution and at least $0.96 \mathrm{~V}$ more negative with respect to the flat band potential in the absence of $\mathrm{O}_{2}$.

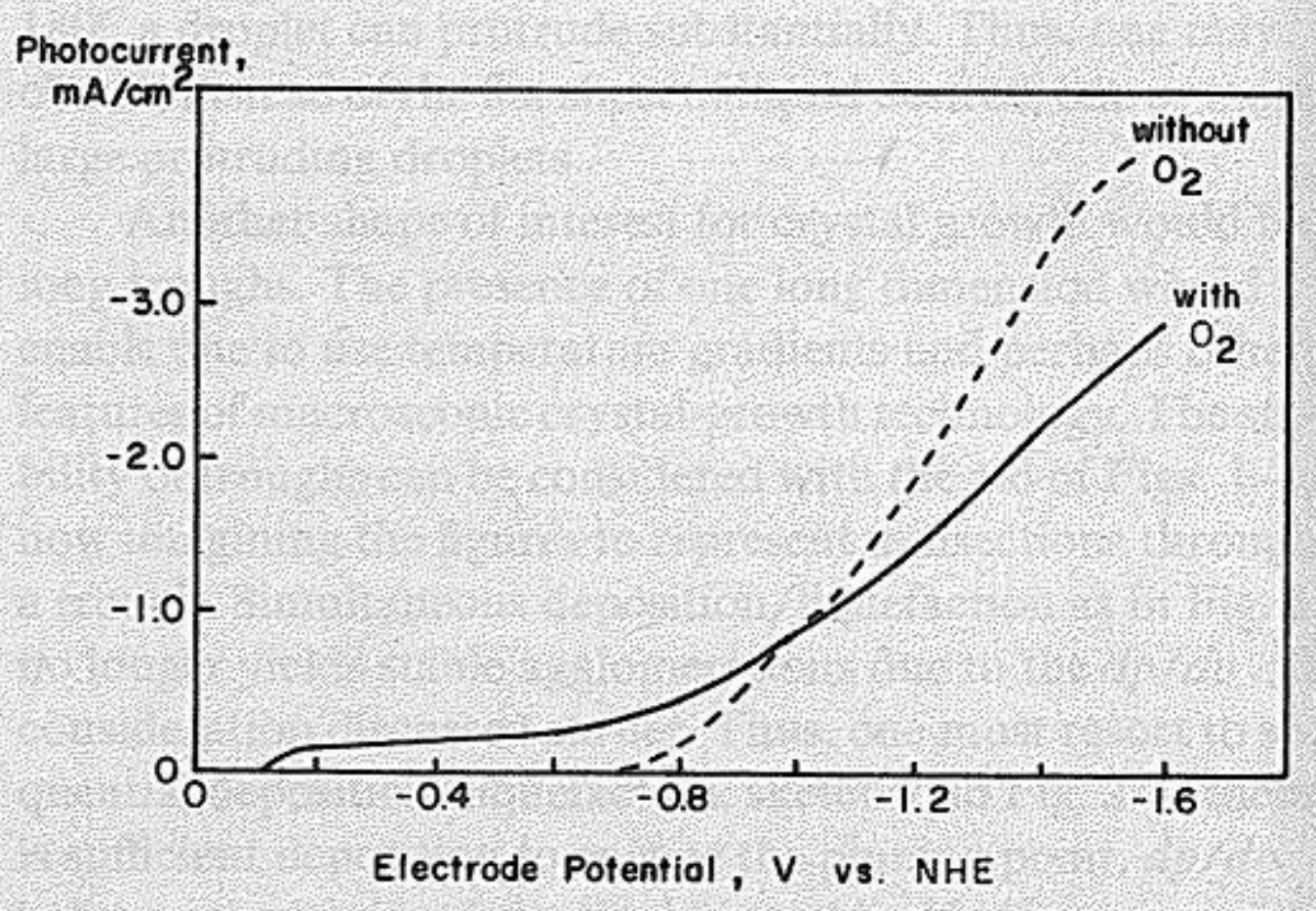

FIG. 1. Potential dependence of photocurrent of $\mathrm{CdTe}$ in $1-\mathrm{N} \mathrm{NaOH}$ with and without $\mathrm{O}_{2}$ in solution. Broken lines: $\mathrm{O}_{2}$ present; continuous lines: no $\mathrm{O}_{2}$ present. 
If one takes the interfacial electron transfer step into account, the photocurrent is given by

$$
i_{p}=\sum_{i=l}^{n} i_{p, i} \alpha \sum_{i=1}^{n} \int_{0}^{\infty} N_{i}(E) W_{i}(E) G_{i}(E) d E
$$

where $i_{p, i}$ is the photocurrent due to electron transfer to an accepter $i$ (e.g., $\left.\mathrm{H}_{3} \mathrm{O}^{+}\right), W_{i}(E)$ is the tunneling probability for electron transfer from a semiconductor to the acceptor $i$ in the solution at energy $E$ to $E+d E$, and $G_{i}(E)$ is the number of acceptors of type $i$ in the solution which has levels at energy $E$ to $E+d E$. For the present case,

$$
\begin{aligned}
& i_{p, \mathrm{O}_{2}} \alpha \int_{0}^{E} N_{p}(E) W_{O_{2}}(E) G_{O_{2}}(E) d E \\
& i_{p, \mathrm{H}_{3} \mathrm{O}^{+}} \alpha \int_{0}^{E} N_{p}(E) W_{\mathrm{H}_{3} \mathrm{O}^{+}}(E) G_{\mathrm{H}_{3} \mathrm{O}^{+}}(E) d E
\end{aligned}
$$

and

$$
i_{p}=i_{p, \mathrm{O}_{2}}+i_{p, \mathrm{H}_{3} \mathrm{O}^{+}}
$$

where $i_{p, \mathrm{O}_{2}}$ and $i_{p, \mathrm{H}_{3} \mathrm{O}^{+}}$are the photocurrents due, respectively, to the reduction of $\mathrm{O}_{2}$ and $\mathrm{H}_{3} \mathrm{O}^{+}, W_{\mathrm{O}_{2}}(E)$ and $W_{\mathrm{H}_{3} \mathrm{O}^{+}}(E)$ are the tunneling probabilities for electron transfer from the semiconductor to $\mathrm{O}_{2}$ and $\mathrm{H}_{3} \mathrm{O}^{+}$at energy $E$ to $E+d E$, and $G_{\mathrm{H}_{3} \mathrm{O}^{+}}(E)$ are the number of $\mathrm{O}_{2}$ and $\mathrm{H}_{3} \mathrm{O}^{+}$levels in solution at energy $E$ to $E+d E$, respectively. Let it be assumed that all excited electrons arriving at the surface have an energy at the bottom of the conduction band. ${ }^{7}$ If the entire (potential difference) p.d. occurs in the semiconductor,

$i_{p}=i_{p, \mathrm{O}_{2}}+i_{p, \mathrm{H}_{3} \mathrm{O}^{+}} \alpha j_{p}(V)\left(W_{\mathrm{H}_{3} \mathrm{O}^{+}} G_{\mathrm{H}_{3} \mathrm{O}^{+}}+W_{\mathrm{O}_{2}} G_{\mathrm{O}_{2}}\right)$,

where $j_{p}(V)$ is the number of photoexcited electrons arriving at the semiconductor surface when the potential is $V, W_{\mathrm{H}_{3} \mathrm{O}^{+}}$ and $W_{\mathrm{O}_{2}}$ are the tunneling probabilites for electron transfer to $\mathrm{H}_{3} \mathrm{O}^{+}$and $\mathrm{O}_{2}$, respectively, at the energy of the bottom of the conduction band, and $G_{\mathrm{H}_{3} \mathrm{O}^{+}}$and $G_{\mathrm{O}_{2}}$ are the number of $\mathrm{O}_{2}$ and $\mathrm{H}_{3} \mathrm{O}^{+}$levels in the double layer at the energy of the bottom of the conduction band. If the potential difference is entirely in the semiconductor, $W$ and $G$ do not depend on the electrode potential, because the energy level of the bottom of the conduction band at the surface is pinned. ${ }^{1}$ Hence the photocurrent-potential curve should be qualitatively similar to the $j_{p}-V$ curve of Fig. 2.

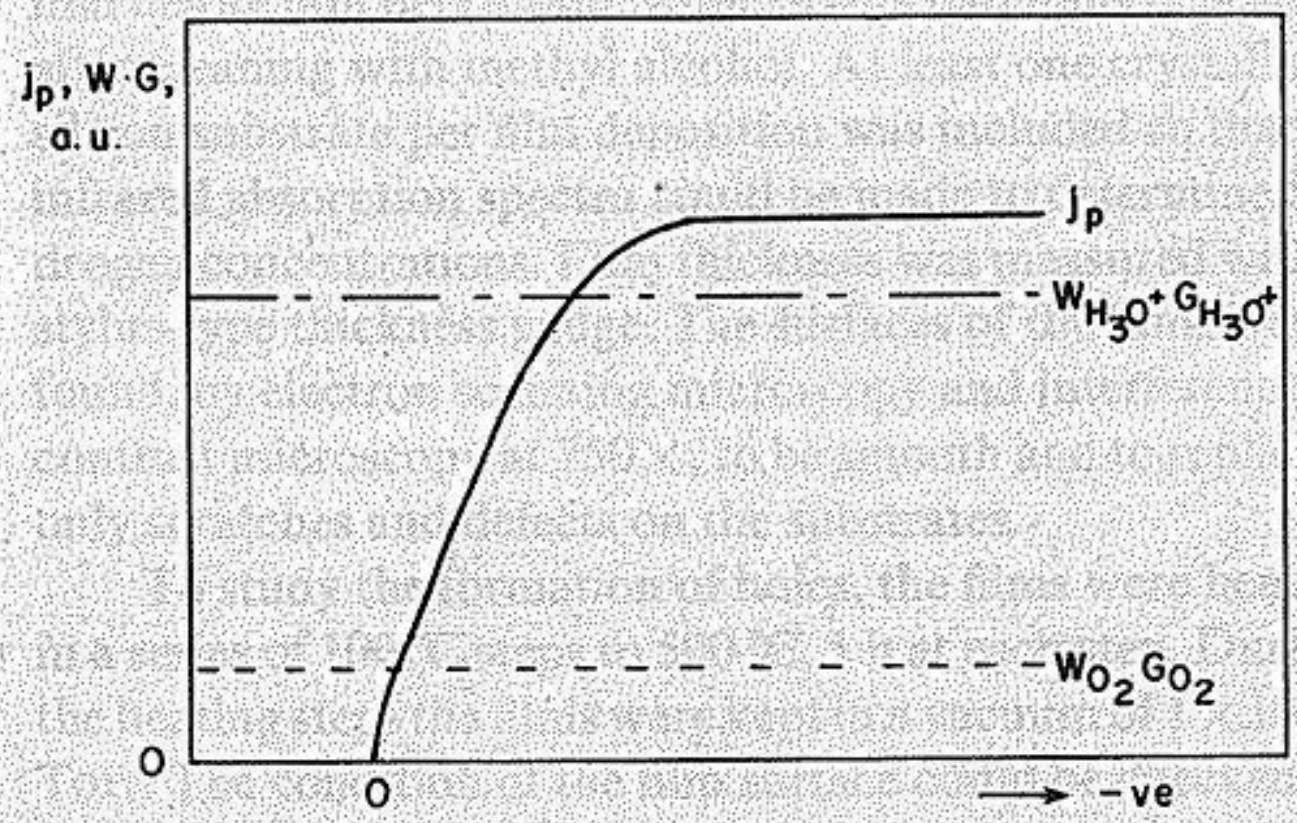

V vS. Flat Band Potential

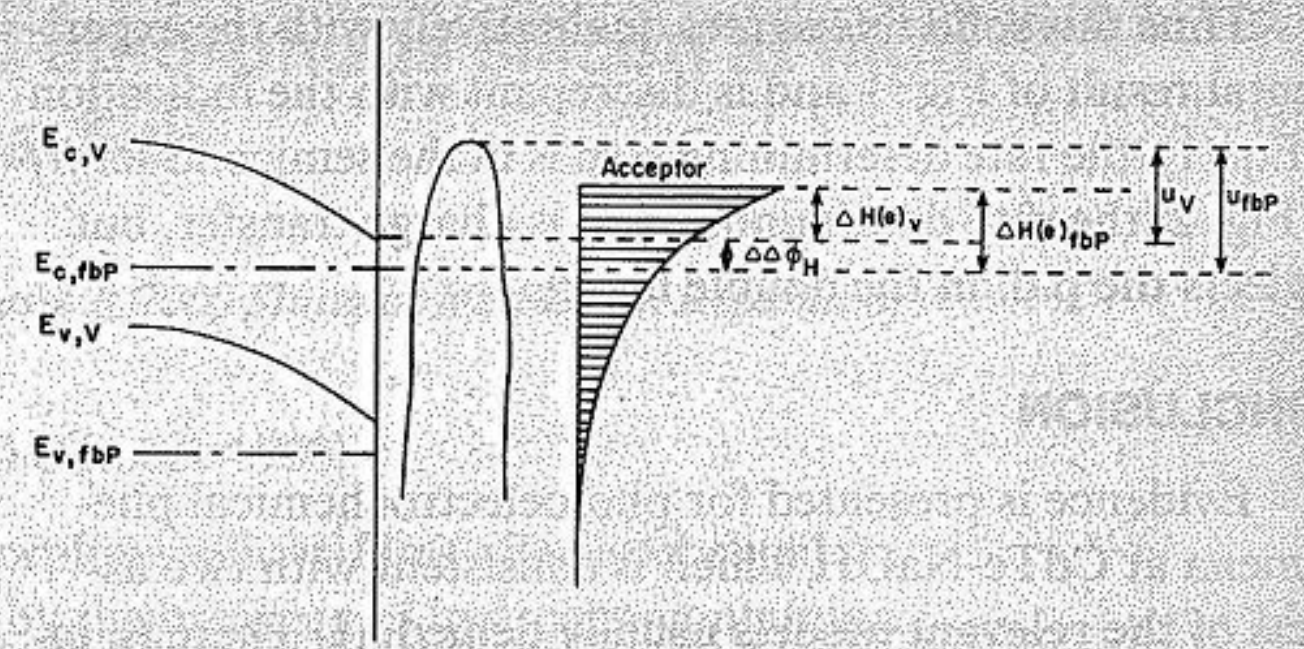

FIG. 3. Schematic diagram of energy distribution at semiconductor-solution interface. Notation in text.

However, if a potential drop in the double layer in the solution is taken into account, this is tantamount to a potential dependence of $W$ and $G$, the values of which will increase until they correspond to the ground state of the acceptor concerned (the level of which has maximum width in Fig. 3).

The situation is to be seen in Fig. 3. The bottom of the conduction and the top of the valence band at the surface have now moved from their pinned positions by $\Delta \Delta \phi_{H}$, which represents that part of the total potential difference at the interface which is in the Helmholtz double layer in the solution. At least in cathodic processes at the semiconductor-solution interface, electrons have to penetrate an "energy barrier", shown in Fig. 3 as a parabola. The height at which the eletrons penetrate the barrier changes (referred to the top of the barrier) from $U_{f b p}$ and $U_{v}$ thus making the barrier lower and hence easier to penetrate. The corresponding energy distances between the ground state of the acceptor changes from $\Delta H(e)_{f b p}$ to $\Delta H(e)_{v}$, thus making a larger number of acceptor levels available in solution.

The behavior of the various entities affecting the current is shown in Fig. 4, where the current is in arbitrary units a.u. The availability of the electrons inside the semiconductor is given by $j_{p}$ and represented by - - - - The lower set of dotted lines refers to $W_{\mathrm{O}_{2}} G_{\mathrm{O}_{2}}$ for electron transfer to $\mathrm{O}_{2}$ and the higher one, that the $\mathrm{H}_{3} \mathrm{O}^{+}$. The result [Eq. (4)] for $i_{p}$ is the solid line.

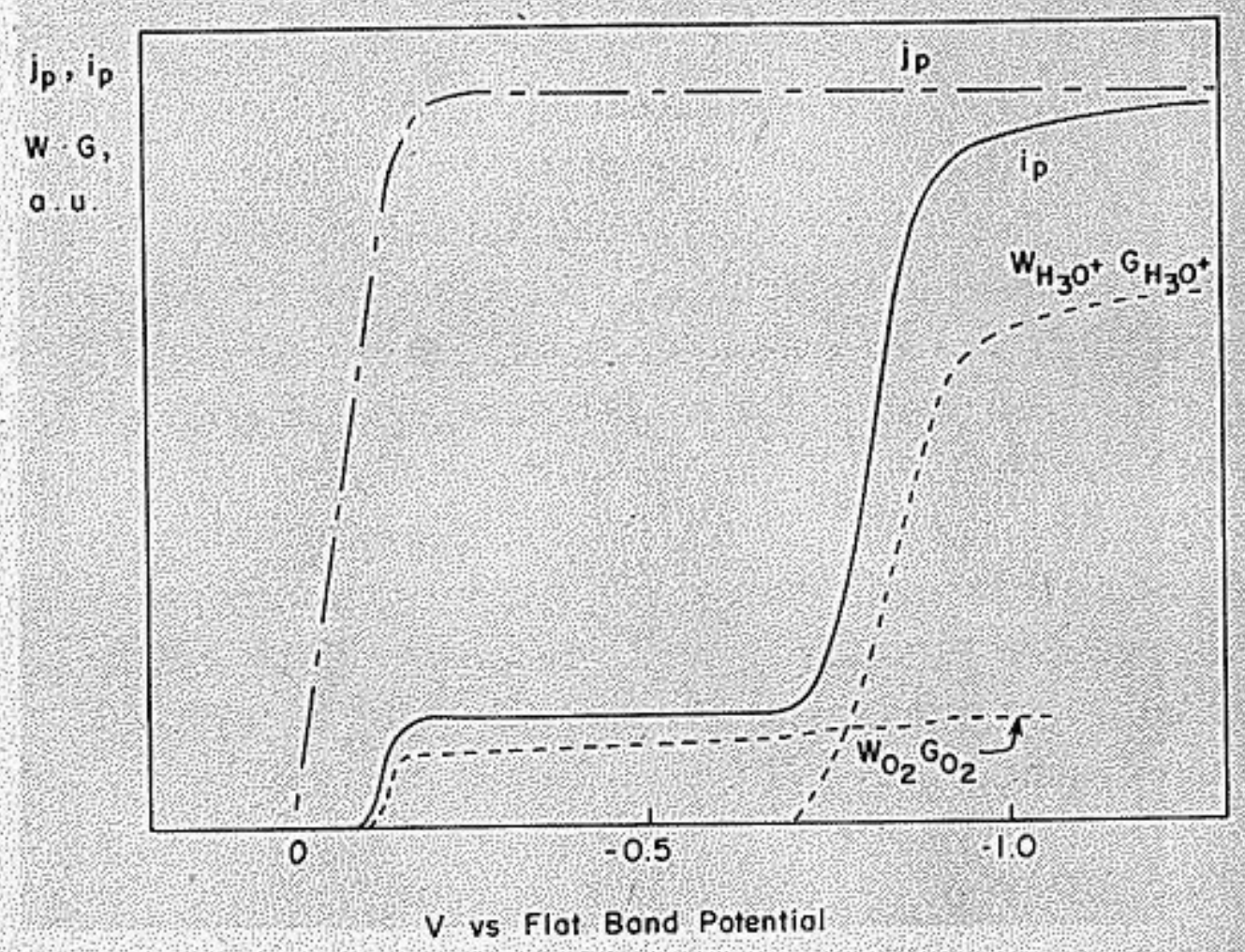

FIG. 4. Potential dependence of $j_{p} W_{\mathrm{O}_{2}} G_{\mathrm{O}_{2}}$, and $i_{p}$. Notation in text. 
Thus this solid line agrees qualitatively with the experimental result of Fig. 1 and is discrepant with the indication of Eq. (1) (the rate determining step is in the semiconductor) or Fig. 2 (the rate determining step is charge transfer, but neglects the p.d. in the double layer).

\section{CONCLUSION}

Evidence is presented for photoelectrochemical phenomena at $\mathrm{CdTe}-\mathrm{NaOH}$ which is consistent with two aspects of the relevant models usually denied: (1) There is, in the system concerned, a significant part of the potential difference at the interface which extends to the Helmholtz layer in solution. (2) Electron transfer across the semiconductorsolution interface affects the net photoelectrochemical rate.

\section{ACKNOWLEDGMENTS}

One of us (K.U.) thanks the Flinders University of South Australia, where the experimental part of this work was carried out, for a scholarship. We also wish to thank $\mathbf{H}$. Kimura of the Mitsubishi Electric Company, Ltd. for a donation of a CdTe single crystal and Dr. K. Ohashi for his help in the preparation of the electrode.

${ }^{1}$ Work published up to 1978 is reviewed by K. Rajeshwar, P. Singh, and J. Dobow, Electrochim. Acta 23, 1117 (1978).

${ }^{2}$ M. A. Butler, J. Appl. Phys, 48, 1914 (1977).

${ }^{3}$ A. K. Ghosh and H. P. Maruska, J. Electrochem. Soc. 124, 1516 (1977).

${ }^{4}$ R. H. Wilson, J. Appl. Phys. 48, 4292 (1977).

5J. O'M. Bockris and K. Uosaki, Adv. Chem. Ser. 163, 33 (1977).

'J. O'M. Bockris and K. Uosaki, J. Electrochem. Soc. 125, 223 (1978).

${ }^{7}$ J. O'M. Bockris and K. Uosaki, J. Electrochem. Soc. 124, 1348 (1977).

${ }^{8}$ A. Hamnett and M. P. Dare-Edwards, J. Electroanal. Chem. 105, 1283 (1979).

${ }^{9}$ H. Gerischer, Adv. Electrochem. Eng. 1, 129 (1961). 